\title{
AIAA 2002-1675 Bell-Curve Genetic Algorithm for Mixed Continuous and Discrete Optimization Problems
}

Rex K. Kincaid

College of William and Mary, Williamsburg, VA 23187-8795

Michelle Griffith

College of William and Mary,

Williamsburg, VA 23187-8795

Ruth Sykes

College of William and Mary, Williamsburg, VA 23187-8795

Jaroslaw Sobieszczanski-Sobieski

NASA Langley Research Center, M/S 240, Hampton, VA 23681, AlAA member

\section{3rd AIAA Structures, Structural Dynamics and Materials April 22-25, 2002/Denver, CO}




\title{
Bell-Curve Genetic Algorithm for Mixed Continuous and Discrete Optimization Problems
}

\author{
Rex K. Kincaid* \\ College of William and Mary, Williamsburg, VA 23187-8795 \\ Micllelle Griffith ${ }^{\dagger}$ \\ College of William and Mary, Williamsburg, VA 23187-8795 \\ Ruth Syke ${ }^{\ddagger}$ \\ College of William and Mary, Williamsburg. VA 23187-8795 \\ Jaroslaw Sobies\%c/anski-Sobieski \\ NASA Langley Research Center, M/S 240, Hampton, VA 23681, AIAA member
}

\section{Introduction.}

This paper is the next installment in a series (Sobieszczanski-Sobieski et al. 1998, Fincaid et al. 2000, 2001a,b and Plassman and SobieszczanskiSobieski 2000) that has introduced a variant of the Genetic Algorithm in which the reproduction mechanism was modified to base it on the Gaussian probability distribution, the bell curve. The bell-curve based ( $\mathrm{BCB}$ ) heuristic procedure, first presented in Sobieszczanski-Sobieski, Laba, and Kincaid (1998), is similar in spirit to Evolutionary Search strategies (ESs) and Evolutionary Programming methods (EPs) but has fewer parameters to adjust. In SobieszczanskiSobieski et al. (1998) BCB was tested on a structural design optimization problem. The quality of solutions generated were verified by comparing $B C B$ solutions to ones generated by a standard nonlinear programming technicque. No attempt was made to analyze the sensitivity of the BC.B parameters. hincaid, Weber and Sobieszczanski-Sobieski (2000) provide a preliminary investigation into $\mathrm{BC} B \mathrm{~B}$ parameter selection as well as document improvements in the performance of BC.B. Computational results for continuous, discrete and mixed continuous and discrete design optimization problems with constraints is reported. Further experiments with BCB for purely discrete op-

*Department of Mathematics, The first and second authors gratefully acknowledge the support of of NASA-Langley Research Center-VAG-1-20TT

${ }^{\dagger}$ Department of Computer Sicience

¥Department of Computer Science

SSenior Research Scientist

Copyright $\odot 2002$ by the American Tnstitute of Aeronautics and Astronautics. Inc. To copyright is asserted in the Inited States under Title 17, ${ }^{-}$.S. Code. The 1 . S. Government has a royaltyfree license to exercise all rights under the copyright claimed herein for Governmental Purposes. Nll other rights are reserved by the copyright owner. timization problems is provided in Kincaid, Weber and Sobieszczanski-Sobieski (2001a). Plassman and Sobieszczanski-Sobieski (2000) implement and test a parallel version of $B C B$ for continuous optimization problems.

The new contribution to be presented in this installment is an extension to applications that encompass a mix of continuous and quasi-discrete, as well as truly-discrete applications. The extension combines a definition of the distance between the parent designs in the space that comprises discrete and continuous variables with application to optimization of statically indeterminate structures in which the order of design variables, e.g. the type of the cross-section, is essential. As expected, the algorithm that accommodates the order information produces better results. In addition, we provide a comparison allowing sampling from the tails of a discrete normal distribution versus a standard mutation scheme. Adding sampling from the tails brings the continuous and discrete versions of $\mathrm{BC} B \mathrm{~B}$ into agreement. Moreover, given the same computing resources, we show that sampling from the tails of the discrete normal leads to higher quality solutions than the standard genetic algorithm mutation approach.

\section{Background Information.}

'To illustrate the connection between BC:B, ESs and EPs we define our mutation, recombination, and selection mechanisms in ES and EP terms. A new generation in our approach is selected exactly the same as a $(\mu+\mu)$-ES. The recombination mechanism is similar to the extension of the intermediate recombination if the weights are required to sum to unity. Consider the line through two n-dimensional parent vectors $\vec{P}_{1}$ and $\vec{P}_{2}$ selected for mating as in Figure 1. Figure 1 depicts a design space simplified to three 
dimensions (three design variables). First, determine the weighted mean $\vec{M}$ of these two vectors where the weights are given by the fitness of each parent. Next, sample from a normal distribution $N\left(0, \sigma_{m}\right)$. The resulting point $\vec{B}=\vec{M}+\left|\vec{P}_{2}-\vec{P}_{1}\right| * N\left(0, \sigma_{m n}\right)$ is the child, prior to mutation. Note that in the extended version of the intermediate recombination method when the weights sum to one the unmutated child can lie anywhere on the line segment between the two parents. In our approach, rather than picking weights arbitrarily, the selection is governed by a normal distribution and the fitness weighted average of the parents. Moreover, $\vec{B}$ is not restricted to lie on the line segment $\overline{P_{1} P_{2}}$. Mutation ensues by first generating a radius $r$ for an $n-1$ dimensional hypersphere. The radius is a realization from a $N\left(0, \sigma_{r}\right)$. Typically $\left(\sigma_{r}>>\sigma_{m}\right)$. Finally the mutated child $\vec{C}$ is selected by sampling uniformly on the surface of the $n-1$ dimensional hypersphere. Since the child can lie anywhere on the surface the effect is similar to the rotated angle portion of an ES or EP (in $n-1$ rather than $n$ dimensions). However, we do not allow the hypersphere to be stretched (or shrunk) along any of its axes as is the case for an ES and EP with non-identical $\sigma_{i}$. We call our procedure a Bell-Curve Based evolutionary optimization algorithm (BCB). Figure 1 is a 3-dimensional view of $\mathrm{BC} B$.

In the case of constraints (or simple bounds) we make two adjustments to $\mathrm{BCB}$. If the point $\vec{B}$ violates a constraint then we select another candidate for $\vec{B}$ until we obtain a feasible one. That is, we sample from $N\left(0, \sigma_{m}\right)$ until $\vec{B}=\vec{M}+\left|\vec{P}_{2}-\vec{P}_{1}\right| * N\left(0, \sigma_{m}\right)$ is feasible. If the child $\vec{C}$ that is produced is infeasible, we translate the components of $\vec{C}$ to the closest constraint. For example, if all variables are to lie within $[1,10]$ and if $\vec{C}=(0.8,2.0,11.9)$, the child would become $(1.0,2.0,10.0)$.

In addition to the general description of $\mathrm{BCB}$ above there are several specific details of note. Instead of a roulette wheel selection scheme for selecting parents we implement Baker's (1987) stochastic universal sampling. In doing so we eliminate the natural bias, noted by Baker (1987), in roulette wheel selection. Although BCB's performance may be improved if knowledge of the underlying feasible region's topology is included in the selection of the initial population, here we generate the initial population randomly (uniformly). There are four parameters that must be chosen to optimize a continuous unconstrained function: population size, number of generations, $\sigma_{m}$, and $\sigma_{r}$. Kincaid et al. $(200 \mathrm{lb})$ provide an investigation into $\mathrm{BC} \mathrm{B}$ parameter selection. We make use of their observations in this manuscript.

First, scaling with respect to the design variables is critical. BCB skews the search space towards design variables of small dimension during recombination. Hence, the search space should be scaled so that all design variables are of similar magnitude. Two normal distributions drive $\mathrm{BC} B-N\left(0, \sigma_{m}\right)$ and $N\left(0, \sigma_{r}\right)$. As we seek to sample along the line through the two parents, $\vec{P}_{1}$ and $\vec{P}_{2}$, we form $\vec{B}=\vec{M}+\left|\vec{P}_{2}-\vec{P}_{1}\right| * N\left(0, \sigma_{m}\right)$. Computational experience in Kincaid et al. (2001b) showed that $\sigma_{m}$ should have a value near 1.0. Intuitively this makes sense since $N\left(0, \sigma_{m}\right)$ serves to inflate or deflate the value of the distance between the parents. Next, to mutate $\vec{B}$ we sample uniformly on the surface of an $n-1$ dimensional hypersphere with radius sampled from our second normal distribution, $N\left(0, \sigma_{r}\right)$. Here, the value of $\sigma_{r}$ is expected to be larger than 1.0 to allow for the possibility of hyperspheres of larger radii. Lnfortunate]y there is no problem specific data from which we can pick a baseline value for the radius of this hypersphere. $N\left(0, \sigma_{r}\right)$ must represent both the unknown radius baseline as well as its normal deviation. As expected, Kincaid et al. (2001b) found that $\sigma_{r}$ should be much larger than $\sigma_{m}$. Below is an outline of the BCB procedure for continuous optimization problems.

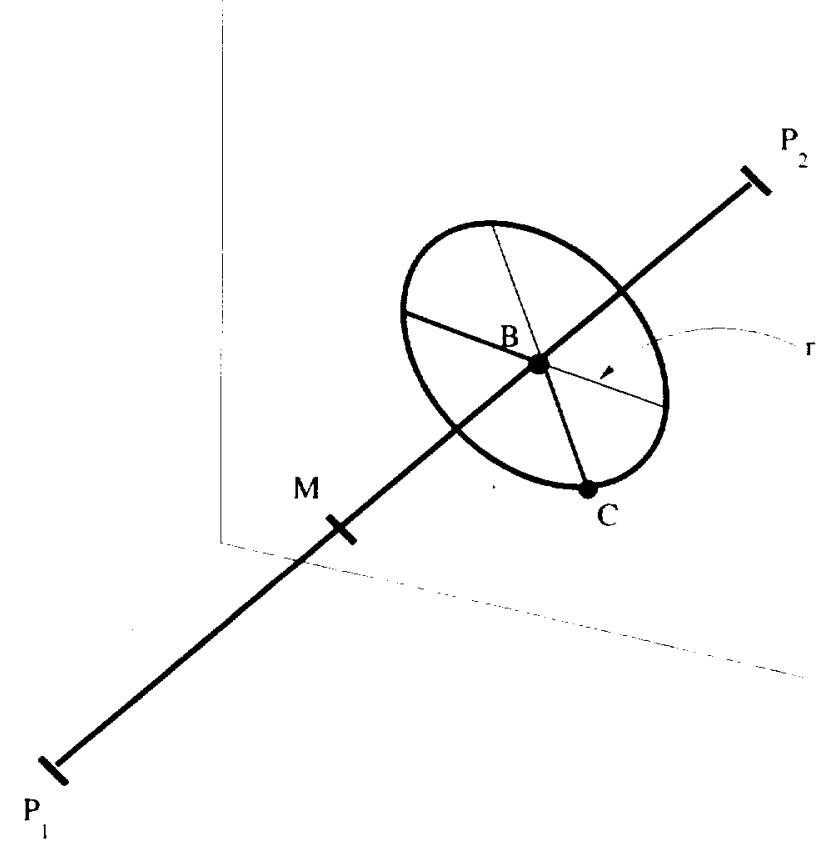

Fig. 1. BC'B Geometrical Construct in 3D Space

Initialize $\mathrm{BC} \mathrm{B}$ by choosing a population size, $\mu$, a maximum number of generations, num $g \in n s, \sigma_{r}$ and $\sigma_{m}$. Scale the search space so that all design variables are of similar magnitude. Randomly generate $\mu$ individuals.

Repeat num-gens times

Repeat $\mu$ times 
Select two parents, $\vec{P}_{1}$ and $\vec{P}_{2}$, from the current population using Baker's stochastic universal sampling.

Calculate $\vec{M}$ the fitmess weighted mean of the parents.

Generate $\vec{B}=\vec{M}+\left|\vec{P}_{2}-\vec{P}_{1}\right| * N\left(0, \sigma_{m}\right)$, the child prior to mutation.

(If $\vec{B}$ violates a constraint sample from $N\left(0, \sigma_{m}\right)$ until a feasible $\vec{B}$ is found.)

Generate $\vec{C}$ the mutated child. Sample from $N\left(0, \sigma_{r}\right)$ to determine $r$, the radius of a hypersphere centered at $\vec{B}$. Sample uniformly on the surface of this hypershere. The resulting point is $\vec{C}$. (If $\vec{C}$ is infeasible we translate the violated components of $\vec{C}$ to lie on the boundary of the violated constraint.)

End Repeat

Keep best $\mu$ of parents and children as parents of next generation

End Repeat

BC,B was originally designed to find high quality solutions to continuous optimization problems. In order to solve mixed continuous and discrete variable optimization BC.B must also be able to optimize purely discrete problems as well. Our first experiment in this regard was to simply round continuous $B C B$ solutions to the nearest discrete value (see Kincaid et al. 2000) for a 2-member hub design problem. This approach is known to work fairly well for quasi-discrete variables, that is, the variables have a continuous physical interpretation but may be implemented only by choosing from a discrete set of options. The thickness of commercially available sheet metal is an example. In contrast, the variables may be truly discrete. Choosing between propellers or jet engines in an aircraft design optimization problem is an example. The rounding approach is totally inapplicable for truly discrete variables. Consequently, in the truly discrete setting there is no obvious analog to a line segment connecting parents. I)efining an underlying geometry via a lattice is possible but our approach is to define an artificial neighborhood structure.

The notion of a neighborhood we use rests on a definition of distance between objects in a finite set. 'This idea is not new. In particular, kelly et al. (1994) use a similar measure in their diversification strategies in tabu search. To the best of our knowledge there is no reference to this in the evolutionary strategy literature. To be generic, consider an example of three objects, each possessing three attributes. Figure $2 \mathrm{~b}$ provides such an example in which the attributes are letters chosen from a list of six letters (Figure ?a). Initially we assume that the order of the attributes in the olject does not matter. We will remove this assumption later. Now, consider object $\beta$ and ask the question: "How many letters must be changed to make $B$ identical to $\alpha ? "$. By inspection, the answer is three. The answer to the same question for $\gamma$ is two. Finally to change $;$ to $\beta$ we need change only one letter. Ol)viously, the above questions may be reversed without changing the answers. That is, the transformation of $\alpha$ to $\gamma$ also requires two letter changes. We now place $\alpha, \beta$, and $\gamma$ on a numerical axis (Figure 2c). Choosing $\alpha$ arbitrarily as a reference at 0 , then the locations of $\gamma$ and $\beta$ are 2 and 3 respectively. That is, each of these objects is placed at the number of attribute changes required to transform it to the reference object ( $\alpha$ in this case).

a) $A$ B C D E F

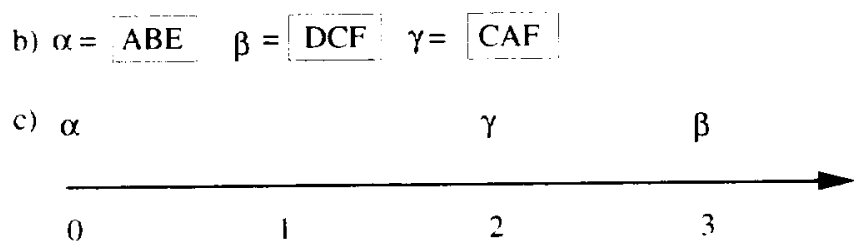

Figure 2. Distance between members of a discrete set

A definition of a distance between objects that are identified by attributes chosen from a finite set emerges from the example in Figure 2.

- The distance between two objects characterized by discrete attributes drawn from the same set is equal to the number of attrilutes that must be changed in one object so that it is identical to the second object in terms of its attributes.

'The distance defined above might also be interpreted as the dissimilarity between two objects. In this interpretation, the null distance corresponds to a complete similarity (identical attributes). The maximum distance, the total number of attributes in the object (three in Figure 2), signifies a complete dissimilarity (no attributes in common). Note that the properties of the similarity and dissimilarity are mutually complimentary - their measures add to the number of attributes.

Now consider the case when the order of attributes in the object does matter. To illustrate the consequence, consider a transformation of $;$ to $\alpha$. In addition to replacing the letters $C$ and $F$ with $B$ and $E$ respectively, one needs to change the position of $A$ in $?$ from position 2 to position 1. Counting the two letter replacements and the position exchange results in $i$ to $\alpha$ distance of 3 . In addition to constructing the single transformations, such as $\gamma$ into $\alpha$, we also want to 
sample among all the objects at a prescribed distance * between the two objects. Kincaid et al. (2000) experimented with discrete problems in which the order of the attributes does NOT matter. In Section 4 we will provide computational experience for problems in which the attribute order does matter.

The tacit assumption in the previous examples is that the number of attribute positions in each object are the same. Although this need not be the case in general, it will be true for the problems we study here. Given the aforementioned distance definition we can now formally describe a discrete version of $\mathrm{BC} B$. We describe the case when the positions of the attributes do matter since that is easier to explain. The symbols $P_{1}, P_{2}$, and $C$ (two parents and the resulting child) correspond to $\alpha, \beta$, and $\gamma$ respectively in the previous discussion. Let $k$ denote the number of attribute positions in $P_{1}$ and $P_{2}$, let $n$ denote the number of possible attribute values $(n>k)$, and let $r$ denote the number of attributes that are dissimilar between $P_{1}$ and $P_{2}$.

- Step 1. Place $P_{1}$ and $P_{2}$ on a numerical axis ranging from 0 to the number of dissimilar components between $P_{1}$ and $P_{2}$. Without loss of generality, let $P_{1}$ serve as the reference point at 0 on this numerical axis.

- Step 2. ('reate a truncated discrete normal distribution on the axis described in step 1. 'The distribution is centered on a point $\boldsymbol{M}$ whose location may be at the midpoint between $P_{1}$ and $P_{2}$ or it may be shifted toward the fittest parent. The distribution is truncated at $P_{1}$ and $P_{2}$. The parameter $\sigma_{n}$ must be chosen by the user.

- Step 3. Let $\varepsilon$ denote the sample value chosen from the distribution in step '2. Place the child $B$ at a distance $\mathrm{from} P_{1}$ on the numerical axis between $P_{1}$ and $P_{2}$.

- Step 4. Reorder the attributes of $P_{1}$ and $P_{2}$ so that the $k-r$ attributes in common appear in the last $k-r$ attribute positions. Assign the remaining $r$ attributes of $P_{1}$ and $P_{2}$ randomly to the first $r$ attribute positions respectively within $P_{1}$ and $P_{2}$. Label these reordered parents $\bar{P}_{1}$ and $\bar{P}_{2}$. Construct a child $B$ of $P_{1}$ and $P_{2}$ as follows. First, set $B=\bar{P}_{2}$. 'Then re-assign the attributes contained in the first $r-$ positions of $P_{1}$ to the first $r-c$ attribute positions of $B$.

- Step 5. Construct the final child $C$ from $B$ by randomly mutating $B$. That is, with a small probability of occurrence, $p$, randomly (uniformly) replace any attribute of $B$ with any of the allowed attribute values.

In addition to experiments in which the attribute order does matter we will also explore the utility of the truncated normal. In order to make the discrete version of $B C B$ closer in spirit to the continuous one it would be nice to replace Step 5 above with sampling in the tails of the discrete normal distribution.

Lastly, we describe how these two algorithms interact when we solve mixed continuous and discrete optimization problems. we treat the discrete and continuous variables as a single collection of decision variables. Suppose that our design structure has $b$ beams, and that there are $t$ possible beam types. Further suppose that the cross-section of each beam type can be described by $n$ contimuous variables. 'Then our solution is a vector $\vec{x}$ that is a concatenation of $b$ vectors of the form

$$
\left(x_{11}, \ldots, x_{1 n} ; x_{21}, \ldots, x_{2 n} ; \ldots ; x_{t 1}, \ldots, x_{t m}\right)
$$

with one such vector for each beam. Here, $x_{i j}$ represents the $j$ th cross-section decision variable of the $i$ th beam type. Thus, a solution will describe not just $b$ beams, but $b * t$ beams. A second vector $\left(y_{1}, y_{2}, \ldots, y_{b}\right)$ indicates the chosen set of beam types, where $y_{i}$ is in the integral range of $[1, t]$. Hence, the value of $y_{i}$ indicates which beam type we have chosen for beam $i$. Given two parents of the form described above, continuous $B C B$ recombines the $\vec{x}$ s and discrete $B C B$ recombines the $\vec{y} \mathrm{~s}$. For further information on other possible ways to solve mixed continuous and discrete optimization problems the interested reader is referred to Kincaid et al. (2001a).

\section{Refinements to Discrete BCB}

In this section we test certain features of discrete BCB. First we examine the two choices for $M$ described in Step 2 of the algorithm. 'That is, should $M$ be the the midpoint between $P_{1}$ and $P_{2}$ or should it be shifted toward the fittest parent. Second, we compare the performance of discrete $\mathrm{BCB}$ as described above to a new version of discrete $B C B$ in which Step 5 is replaced by allowing sampling to occur in the tails of the discrete normal distribution defined in Step 2. That is, the discrete normal distribution defined in Step 2 is no longer truncated. In addition we will limit our attention to problems in which the attribute order does NOT matter.

The test cases for these experiments is a constrained mixed continuous and discrete weighted shape selection optimization problem. We wish to select 5 shapes from a list of three (circle, triangle, and square) or more geometrical shapes. The five positions are numbered in order 1 through 5 . The goal is to choose 5 shapes (possibly with repeats) and the dimensions of those five chosen shapes to minimize the total weighted sum of the perimeters. Each chosen shape's perimeter is weighted by its position in the list: the first shape in the list is weighted by 5 , the second by 4 , the third 
by 3 , and so on. The continuous variable is the radius (r) for the circle, the length of a side (s) for the square and the length of the base (b) for a right isosceles triangle. The constraints are as follows. The total area must be greater than 100 units and each continuous variable has a lower bound of 1.0 and an upper bound of 10.0. The optimal set of shapes is 4 triangles and 1 circle with an oljective value of 8.9 . The circle has the smallest perimeter to area ratio $(2 / \mathrm{r})$ and is chosen for position 5 with $r$ just large enough so that 100 - (sum of the 4 triangles area) is satisfied. Small triangles are chosen for the remaining 4 shapes since its perimeter to area ratio is largest. In an attempt to understand how BCB scales with respect to the number of discrete variables we solve the position dependent shape problem with the addition of regular polygons with $5,6,8$, and 10 sides.

Table 1. Using fitness: 15 reps, 20,000 solutions, 0.04 mutation

\begin{tabular}{|c|c|c|c|c|}
\hline $\begin{array}{c}\# \\
\text { Shapes }\end{array}$ & $\begin{array}{c}\text { Mean } \\
\text { Best Obj }\end{array}$ & $\begin{array}{c}\text { Min } \\
\text { Best Obj }\end{array}$ & $\begin{array}{c}\text { Max } \\
\text { Best Obj }\end{array}$ & $\begin{array}{c}\text { Freq Opt } \\
\text { Shapes }\end{array}$ \\
\hline 3 & 85.9 & 82.9 & 89.1 & $5 / 15$ \\
\hline 4 & 87.4 & 82.9 & 94.3 & $2 / 15$ \\
\hline 5 & 89.7 & 85.3 & 95.3 & $0 / 15$ \\
\hline 6 & 91.2 & 85.1 & 97.1 & $0 / 15$ \\
\hline 7 & 91.0 & 85.2 & 97.6 & $0 / 15$ \\
\hline
\end{tabular}

Table 2. Using midpoint: 15 reps, 20,000 solutions, 0.04 mutation

\begin{tabular}{|c|c|c|c|c|}
\hline $\begin{array}{c}\# \\
\text { Shapes }\end{array}$ & $\begin{array}{c}\text { Mean } \\
\text { Best Obj }\end{array}$ & $\begin{array}{c}\text { Min } \\
\text { Best Obj }\end{array}$ & $\begin{array}{c}\text { Max } \\
\text { Best Obj }\end{array}$ & $\begin{array}{c}\text { Freq Opt } \\
\text { Shapes }\end{array}$ \\
\hline 3 & 85.7 & 82.9 & 89.4 & $6 / 15$ \\
\hline 4 & 86.1 & 82.9 & 90.4 & $5 / 15$ \\
\hline 5 & 89.1 & 83.7 & 93.2 & $1 / 15$ \\
\hline 6 & 93.0 & 85.7 & 98.1 & $0 / 15$ \\
\hline 7 & 90.8 & 83.9 & 96.7 & $0 / 15$ \\
\hline
\end{tabular}

In the above experiment the fitness weighted mean in Step 2 of the algorithm does not perform quite as well as simply using the midpoint of $P_{1}$ and $P_{2}$. The midpoint approach has the min and mean best oljective values in 4 of the 5 cases and identifies more optimal shapes (12) than the fitness approach ( 7 ). Even so the appeal of making use of parent fitness has led us to continue with its use. One reason for doing so is to make the continuous and discrete implementations of $\mathrm{BCB}$ as similar as possible.

The next set of experiments seeks to compare the truncated discrete normal with mutation versus a discrete normal distribution in Step 2 of the algorithm. The table below summarizes these experiments for the discrete normal distribution which can be compared against the results in Tables 1 and 2 . Here we see that the use of tails for the discrete normal is beneficial. 26 optimal shapes are found with the tails and only 12 with the mutation approach. In addition, the mean best objective value for the tails approach wins in 4 of the 5 cases.

Table 3. Discrete Normal with tails: 15 reps, 20,000 solutions

\begin{tabular}{|c|c|c|c|c|}
\hline $\begin{array}{c}\# \\
\text { Shapes }\end{array}$ & $\begin{array}{c}\text { Mean } \\
\text { Best Obj }\end{array}$ & $\begin{array}{c}\text { Min } \\
\text { Best Obj }\end{array}$ & $\begin{array}{c}\text { Max } \\
\text { Best Obj }\end{array}$ & $\begin{array}{c}\text { Freq Opt } \\
\text { Shapes }\end{array}$ \\
\hline 3 & 84.0 & 82.9 & 88.4 & $13 / 15$ \\
\hline 4 & 85.6 & 82.9 & 90.6 & $7 / 15$ \\
\hline 5 & 89.0 & 82.9 & 99.9 & $5 / 15$ \\
\hline 6 & 91.3 & 86.0 & 99.9 & $0 / 15$ \\
\hline 7 & 91.8 & 82.9 & 99.6 & $1 / 15$ \\
\hline
\end{tabular}

\section{Hub Design Problem}

Our standard test problem in SobieszczanskiSobieski et al. (1998), Kincaid et al. (2000) and Kincaid (2001) has been a minimum weight (volume) design of a hub structure also found in Balling and Sobjeszczanski-Sobieski (1994). Each member of the hub structure is an l-beam rigidly attached to the hub, and to the wall, forming a two-dimensional, wheelwith-spokes pattern whose two-beam version is illustrated in Figure 3. The structure load has three components, horizontal and vertical concentrated forces, and a concentrated moment, all acting on the hub in the plane of the structure. Various loading cases (henceforth called "loads") are formed from the above load components. Three loading cases are applied to a ti-beam structure, and two loading case are used in case of a 20 -beam structure to reduce the computing elapsed time. The beam cross-sectional dimensions are the design variables, and the constraint functions reflect the material allowable stress as well as overall and local buckling. The top and bottom flanges of the I-beam are not necessarily of the same dimensions. Hence, the cross-section of each I-beam requires six design variables. Figure 3 illustrates a 2-member hul, problem. Additional details may be found in Padula et al.(1996). The utility of the hub structure as a test case stems from its ability to be enlarged by adding as many members as desired without increasing the dimensionality of the load-deflection equations. These remain 3 by 3 equations for a 2 -dimensional hub structure regardless of the number of members. While analytically simple, the hub structure design space is complex because the stress, displacement and buckling constraints are rich in nonlinearities and couplings among the design variables. Our goal is to extend this problem so that, in addition to choosing the dimensions of the beams so as to minimize the volume of the hub frame, we would also select (among a finite list) the beam type.

As a first step in this direction we consider 3 beam cross-section types - I, circular, and triangular. In all cases the overall length of the beam is held constant amongst the cross-section types. The I-beam has 6 design variables; the circular beam has 2 design vari- 
ables; and the triangular beam has 3 design variables. Our first experiment is with a 6 beam 3 load hub design problem. Each beam has the same nominal length of 120.0 and the following $B$ C.B parameters values:

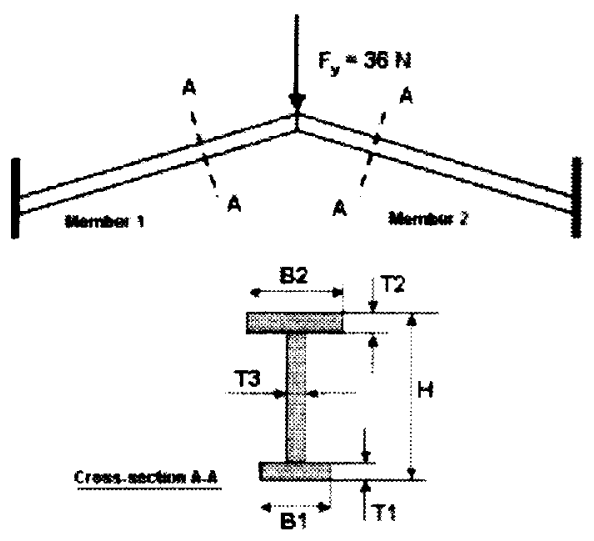

Figure 3. 2-member hub description

$\begin{array}{ll}\text { population size } & : 20 \\ \text { number of generations } & : 1250 \\ \text { replications } & : 50 \\ \text { PENALTY1 } & : 2000.0 \\ \text { PENALTY2 } & : 4000.0 \\ \text { fitness-related mean for discrete } & \\ \text { normal number of swaps }\end{array}$

As a benchmark we force mixed $\mathrm{BCB}$ to choose all I-beams and obtain the following results:

\begin{tabular}{|l|c|c|}
\hline & $\begin{array}{c}\text { Order NOT } \\
\text { Important }\end{array}$ & $\begin{array}{c}\text { Order } \\
\text { Important }\end{array}$ \\
\hline min vol & 576.62 & 554.51 \\
\hline mean vol & 687.81 & $681.0^{\circ}$ \\
\hline s.d. vol & 63.63 & 126.33 \\
\hline
\end{tabular}

The case in which the order of matching elements in the parents matters leads to slightly better results. This trend has been borne out in all of computational experience although the order is important approach seems to require more generations to converge. We expect this is due to the larger neighborhood of feasible solutions when order counts.

With the penalties shown above, the maximum constraint violations occurred as follows:

\begin{tabular}{|l|c|c|}
\hline & $\begin{array}{c}\text { Order NOT } \\
\text { Important }\end{array}$ & $\begin{array}{c}\text { Order } \\
\text { Important }\end{array}$ \\
\hline mean max. violation & -0.00039 & -0.0085 \\
\hline s.d. max. violation & 0.0027 & 0.0596 \\
\hline
\end{tabular}

The following table lists the results when mixed $\mathrm{BCB}$ is allowed to choose one of three possible shapes (Ibeam: 1, circle: 2 , or triangle:3) for each of the six beams when the order does not matter in computing the distance between discrete components.

Table 4: Frequencies of designs chosen (order does not matter)

\begin{tabular}{|c|c|c|c|c|}
\hline Design & $\#$ & $\begin{array}{c}\text { Min } \\
\text { Best Vol }\end{array}$ & $\begin{array}{c}\text { Mean } \\
\text { Best Vol }\end{array}$ & $\begin{array}{c}\text { Std Dev } \\
\text { Best Vol }\end{array}$ \\
\hline $1,1,1,2,2,2$ & 1 & 456.90 & 456.90 & - \\
$1,1,2,1,2,2$ & 1 & 711.10 & 711.10 & - \\
$1,2,1,3,2,2$ & 1 & 448.84 & 448.83 & - \\
$1,2,2,2,1,2$ & 1 & 404.51 & 404.51 & - \\
$1,2,2,2,2,2$ & 2 & 383.29 & 38.3 .29 & 0.001 \\
$1,3,2,2,2,2$ & 1 & 429.78 & 429.78 & - \\
$1,3,2,2,3,2$ & 1 & 464.50 & 464.50 & - \\
$2,1,1,2,2,2$ & 1 & 416.85 & 416.85 & - \\
$2,1,2,2,2,2$ & 1 & 462.20 & 462.20 & - \\
$2,1,2,2,2,3$ & 1 & 592.75 & 592.75 & - \\
$2,1,2,3,2,2$ & 1 & 447.8 .3 & 447.83 & - \\
$2,1,3,2,2,1$ & 1 & 477.77 & 477.77 & - \\
$2,2,2,1,2,2$ & 1 & 417.72 & 417.72 & - \\
$2,2,2,1,3,2$ & 1 & 464.91 & 464.91 & - \\
$2,2,2,2,1,1$ & 1 & 461.79 & 461.79 & - \\
$2,2,2,2,1,2$ & 1 & 383.20 & 38.3 .20 & - \\
$2,2,2,2,2,1$ & 1 & 471.11 & 471.11 & - \\
$2,2,2,2,2,2$ & 8 & 361.91 & 365.98 & 10.51 \\
$2,2,2,2,2,3$ & 1 & 426.61 & 426.61 & - \\
$2,2,2,2,3,2$ & 2 & 401.99 & 422.61 & 20.62 \\
$2,2,2,3,2,1$ & 1 & 533.66 & 533.66 & - \\
$2,2,2,3,2,2$ & 2 & 416.74 & 447.42 & 30.68 \\
$2,2,3,2,2,2$ & 2 & 401.51 & 401.51 & 0.002 \\
$2,3,2,2,2,2$ & 2 & 424.75 & 426.89 & 2.14 \\
$2,3,2,2,2,3$ & 1 & 576.26 & 576.26 & - \\
$2,3,3,2,2,2$ & 1 & 541.52 & 541.52 & - \\
$2,3,3,2,2,2$ & 1 & 502.52 & 502.52 & - \\
$3,1,2,2,2,2$ & 1 & 489.31 & 489.31 & - \\
$3,1,3,3,2,2$ & 1 & 541.69 & 541.69 & - \\
$3,2,2,2,1,2$ & 1 & 429.18 & 429.18 & - \\
$3,2,2,2,2,2$ & 7 & 401.49 & 401.49 & 1.65 \\
$3,3,2,2,2,2$ & 1 & 527.12 & 527.12 & - \\
\hline
\end{tabular}

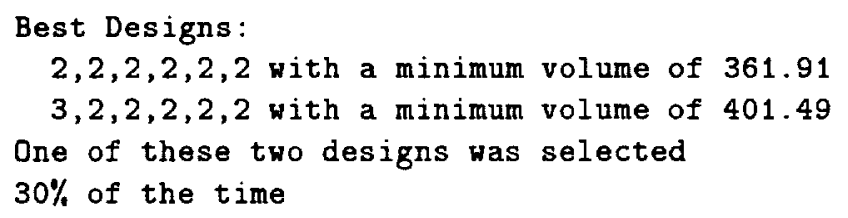

The following table lists the results when mixed $\mathrm{BCB}$ is allowed to choose one of three possible shapes (Ibeam: 1 , circle: 2 , or triangle: 3 ) for each of the six beams when the attribute order does matter in computing the distance between discrete components. 
Table 5: Frequencies of designs chosen (order does matter)

\begin{tabular}{|c|c|c|c|c|}
\hline Design & $\#$ & $\begin{array}{c}\text { Min } \\
\text { Best Vol }\end{array}$ & $\begin{array}{c}\text { Mean } \\
\text { Best Vol }\end{array}$ & $\begin{array}{c}\text { Std Dev } \\
\text { Best Vol }\end{array}$ \\
\hline $1,2,2,1,2,2$ & 1 & 421.07 & 421.07 & - \\
$1,2,2,2,2,2$ & 3 & 383.19 & 383.44 & 0.21 \\
$2,2,1,2,1,2$ & 1 & 404.90 & 404.90 & - \\
$2,2,2,1,1,1$ & 1 & 479.39 & 479.39 & - \\
$2,2,2,1,2,2$ & 3 & 399.77 & 477.93 & 61.39 \\
$2,2,2,2,1,2$ & 2 & $3 \times 6.97$ & 436.16 & 49.19 \\
$2,2,2,2,1,3$ & 2 & 434.42 & 488.10 & 53.67 \\
$2,2,2,2,2,2$ & 22 & 361.91 & 362.00 & 0.15 \\
$2,2,2,2,2,3$ & 1 & 412.54 & 412.54 & - \\
$2,2,2,3,2,1$ & 1 & 527.49 & 527.49 & - \\
$2,2,2,3,2,2$ & 3 & 407.82 & 427.82 & 14.48 \\
$2,2,2,3,3,2$ & 1 & 451.82 & 451.82 & - \\
$2,2,3,2,2,1$ & 1 & 411.57 & 441.57 & - \\
$2,2,2,3,2,2$ & 1 & 517.07 & 517.07 & - \\
$2,3,1,3,2,2$ & 1 & 53.54 & 538.54 & - \\
$2,3,2,1,2,2$ & 1 & 485.36 & 485.36 & - \\
$2,3,2,2,2,2$ & 1 & 410.65 & 410.65 & - \\
$2,2,2,2,3,2$ & 1 & 444.29 & 444.29 & - \\
$3,1,2,2,2,2$ & 1 & 476.3 .3 & 476.33 & - \\
$3.2,2,2,2,2$ & 1 & 423.71 & 423.71 & - \\
$3,2,2,2,2,2$ & 1 & 441.13 & 441.13 & - \\
\hline
\end{tabular}

\section{Best Designs:}

$2,2,2,2,2,2$ with a minimum volume of 361.91

$1,2,2,2,2,2$ with a minimum volume of 383.19

$2,2,2,1,2,2$ with a minimum volume of 386.97

$2,2,2,1,2,2$ with a minimum volume of 399.77

One of these four designs was chosen

$60 \%$ of the time.

With the same penalties as before, the maximum constraint violations occurred as follows:

\begin{tabular}{|l|c|c|}
\hline & $\begin{array}{c}\text { Order NOT } \\
\text { Important }\end{array}$ & $\begin{array}{c}\text { Order } \\
\text { Important }\end{array}$ \\
\hline mean max violation & $\mathbf{- 0 . 0 7 6}$ & $\mathbf{- 0 . 0 6 1 9}$ \\
\hline s.d. max violation & $\mathbf{0 . 0 \times 4 9}$ & 0.06 \\
\hline
\end{tabular}

The above tables indicate the trends we have seen in variety of problem instances. The "order does matter" approach results in fewer, higher quality solutions and the distribution of final solutions is tighter (smaller variance) with a higher quality mean.

Next, we consider a 20 beam 2 load hub design problem. Again all 20 beams are of the same nominal length. The same $B$ C $B$ parameters were used as in the previous experiment except that Penalty 1 was increased to 10,000 and Penalty 2 was increased to 20,000 . With these penalties, the program had a condition added that the maximum constraint violation must be less than $0.0^{\circ}$. Again, as a benchmark, the following results are obtained when mixed $B C \mathrm{C} B$ is allowed only to choose I-beams:

\begin{tabular}{|l|c|c|}
\hline & $\begin{array}{c}\text { Order NOT } \\
\text { Important }\end{array}$ & $\begin{array}{c}\text { Order } \\
\text { Important }\end{array}$ \\
\hline min. vol. & 17089.629 & 17112.098 \\
\hline mean vol. & 18984.661 & $17 \times 69.609$ \\
\hline s.d. vol. & 2051.516 & 1285.047 \\
\hline
\end{tabular}

The use of the "order does matter" definition of discrete distance yields results that are only slightly better with respect to the mean but are significantly better with regard to the variance in the results.

The following table lists the results when mixed $B C B$ is allowed to choose one of three possible shapes (Ibeam: 1 , circle:2, or triangle: 3 ) for each of the six beams.

\begin{tabular}{|l|c|c|}
\hline & $\begin{array}{c}\text { Order NOT } \\
\text { Important }\end{array}$ & $\begin{array}{c}\text { Order } \\
\text { lmportant }\end{array}$ \\
\hline min. vol. & 12865.855 & 13394.102 \\
\hline shapes & $2,2,1,3,2,2,1,3,2,2$, & $2,2,2,2,2,2,1,3,2,1$, \\
& $2,3,2,1,1,2,2,2,2,1$ & $2,2,2,2,2,2,2,2,2,2$ \\
\hline mean vol. & 16140.3399 & 14278.798 \\
\hline s.d. vol. & 1694.326 & 1070.669 \\
\hline
\end{tabular}

Hrre we can see a significant improvement with the use of the "order does matter" discrete distance definition.

Next we increase the number of choices for the beam type (the only discrete variable) from 3 to 6 . The shape choices for each beam are 1:I, 2:circle, 3:triangle, 4:rectangle, $5: \mathrm{L}$, or $6: \mathrm{C}$. This problem was run with all 6 beams having the same nominal length and the following $\mathrm{BCB}$ parameters:

$\begin{array}{ll}\text { population size } & : 20 \\ \text { number of generations } & : 1250 \\ \text { replications } & : 50 \\ \text { PENALTY1 } & : 2000.0 \\ \text { PENALTY2 } & : 4000.0 \\ \text { fitness-related mean for discrete } \\ \text { normal number of swaps }\end{array}$

The experiment was first run for the case when "order does NOT matter" and, of the 50 replications, only the following solutions were repeated:

\begin{tabular}{|c|c|c|c|c|}
\hline Design & $\#$ & $\begin{array}{c}\text { Min } \\
\text { Best Vol }\end{array}$ & $\begin{array}{c}\text { Mean } \\
\text { Best Vol }\end{array}$ & $\begin{array}{c}\text { Std Iev } \\
\text { Best Vol }\end{array}$ \\
\hline $1,2,2,2,2,2$ & 2 & $383.2 \cdot 2$ & 38.3 .34 & 0.12 \\
$2,2,2,2,6,2$ & 2 & 456.41 & 467.58 & 11.17 \\
\hline
\end{tabular}

The range of values for all the solutions was 361.91 $(2.2,2,2,2,2)$ to $977.27(5,6,4,5,2,1)$ with an average value of 533.11 and standard deviation of 119.91 .

The same experiment was repeated for the case when order does matter and, of the 50 replications, only the following solutions were repeated: 


\begin{tabular}{|c|c|c|c|c|}
\hline Design & $\#$ & $\begin{array}{c}\text { Min } \\
\text { Best Vol }\end{array}$ & $\begin{array}{c}\text { Mean } \\
\text { Best Vol }\end{array}$ & $\begin{array}{c}\text { Std Dev } \\
\text { Best Vol }\end{array}$ \\
\hline $2,2,1,2,2,2$ & 2 & 383.20 & 383.32 & 0.12 \\
$2,2,2,2,2,2$ & 6 & 361.91 & 376.94 & 33.22 \\
$2,2,2,2,5,2$ & 2 & 395.20 & 415.09 & 19.89 \\
$2,2,5,2,2,2$ & 2 & 395.20 & 449.81 & 54.61 \\
\hline
\end{tabular}

The range of values for all the solutions was 361.91 $(2,2,2,2,2,2)$ to $1322.02(5,2,6,2,2,2)$ with an average value of 526.35 and standard deviation of 148.02 . If the single outlier value of $132 \% .02$ is discarded, the maximum value is $701.03(1,2,2,2,3,5)$ and the average value over the 50 replications is 507.85 with a standard deviation of 85.75 . In this manner we have a tighter range of values then with the "order does not matter" definition of distance.

Next we consider the 20 beam 2 load problem and use the same $\mathrm{BC} B$ parameters as in our previous 20 beam 2 load case except that 6 shape choices (1:I, 2:circle, 3:triangle, 4:rectangle, $5: \mathrm{L}$, or $6: \mathrm{C})$. are now available. This yielded the following results over 20 replications:

\begin{tabular}{|l|c|c|}
\hline & $\begin{array}{c}\text { Order NOT } \\
\text { Important }\end{array}$ & $\begin{array}{c}\text { Order } \\
\text { Important }\end{array}$ \\
\hline min. vol. & 14949.145 & 14359.097 \\
\hline shapes & $2,4,4,2,2,6,1,2,2,2$, & $4,2,4,5,1,1,4,2,2,4$, \\
& $6,2,2,3,2,3,4,6,4,5$ & $2,3,3,2,5,4,3,2,2,6$ \\
\hline mean vol. & 16827.441 & 15803.128 \\
\hline max. vol. & 19001.340 & 17734.045 \\
\hline shapes & $1,3,4,6,4,6,5,6,2,6$, & $4,5,4,5,4,1,2,1,2,6$, \\
& $5,2,2,5,4,4,2,2,1,1$ & $2,2,1,2,2,1,5,2,6,1$ \\
\hline s.d. vol. & 1233.146 & 901.371 \\
\hline
\end{tabular}

The use of the "order does matter" definition for discrete distance yields results that are both slightly better with respect to the mean and less spread out (smaller standard deviation) over the multiple runs.

\section{Concluding Remarks}

In this manuscript we have examined an extension of $\mathrm{BCB}$ that encompasses a mix of continuous and quasi-discrete, as well as truly-discrete applications. We began by testing two refinements to the discrete version of $\mathrm{BCB}$. The testing of midpoint versus fitness (Tables 1 and 2 ) proved inconclusive. The testing of discrete normal tails versus standard mutation showed was conclusive and demonstrated that the discrete normal tails are better. Next, we implemented these refinements in a combined continuous and discrete BCB and compared the performance of two discrete distance on the hub problem. Here we found when "order does matter" it pays to take it into account.

The computational experiments we have performed lead naturally to additional questions. Will the performance of mixed $B C B$ be improved if $B C B$ is linked with pattern search? Pattern search is a derivative free local search procedure that has been shown to perform well on a variety of nonlinear optimization problems (see Lewis et al. 2000 for details). Previous work (Kincaid et al. 2001b) has shown that BCB coupled with local search procedures works well for the continuous version of $\mathrm{BC} B$. The reasoning is that $\mathrm{BCB}$ does a good job of identifying valleys of interest but takes too long getting to the bottom. Will the same be true in the mixed continuous and discrete case?

Our work here focused on a small scale structural optimization problem for testing the performance of discrete $B C B$. It is natural to wonder if discrete $B C \cdot B$ will stand up against other competitors-tabu search, simulated annealing, and traditional genetic algorithms. $A$ potential scalable problem venue to test this conjecture is the the NK-landscape model. The NK model is due to Kauffman (1989) and is a random energy model similar to a spin glass. It is designed to be tuneable. That is, as $\mathrm{K}$ increases the landscape becomes more rugged. When $K=0$ a highly correlated landscape with a single peak results. At the other extreme, when $K=N-1$ (where $N$ is the number of decision variables) a completely uncorrelated landscape results with very many peaks.

\section{References}

Baker, J.E. (1987). Balancing Diversity and Convergence in Genetic Search. Dissertation, Computer Science Dept. Vanderbilt I niversity, Nashville, TN.

Balling, R..J. and J. Sobieszczanski-Sobieski, "An Algorithm for Solving the System-Level Problem in Multilevel Optimization," ICASE Report No. 94-96 and NASA Contractor Report 195015, December 1994.

Kauffman, S.A. (1989) "Adaptation on Rugged Fitness Landscapes," in D.L. Stein (ed.) Lectures in the Sciences of Complexity, Addison Wesley, Vol. 1, pp. $52 \bar{\imath}-618$.

Kelly, J.P., M. Laguna, and F. Glover (1994) "A Study on Diversification Strategies for the Quadratic Assignment Problem," Computers and Operations Research, Vol. 22, No.8, pp. $8 \times 5-893$.

Kincaid, R.K., Weber, M. and SobieszczanskiSobieski, J. (2000) "Performance of a Bell-Curve Based Evolutionary Optimization Algorithm," Proceedings of the 41st AIAA Structures, Structural Dynamics, and Materials Conference, Atlanta, GA, AIAA 2000$138 \times$.

Kincaid, R.K., Weber, M. and SobieszczanskiSobieski, J. (2001a) "A Bell-Curve Based Algorithm for Mixed Continuous and Discrete Structural Optimization," Proceedings of the 420nd AIAA Structures, Structural Dynamics, and Materials Conference, Seat- 
tle, WA, AIAA 2001-1550.

Kincaid, R.K., Weber, M. and SobieszczanskiSobieski, J., "An Atypical $(\mu+\mu)$ Evolutionary Algorithm," submitted for publication in $2001 \mathrm{~b}$.

Lewis, R.M., Torczon, V. and Trosset, M. (2000). Direct Search Methods: Then and Now. 'lo appear: $J$. of Computational and Applied Mathematics.

Padula, S.L., Alexandrov, N. and (Green, L.L. (1996). MDO Test Suite at NASA Langley Research Center. In: proceedings of the 6th AIAA/NASA/ISSMO Symposium on Multidisciplinary Analysis and Optimization. (pp. 410-420). Bellvue, WA. (See http://fmadwww.larc.nasa.gov/mdob/)

Plassman, J. and Sobieszczanski-Sobieski, J. (2000). Parallel Scalability of Bel]-Curve Based Optimization. Working Paper. NASA-Langley Research C'enter. Hampton, VA 23681 .

Sobieszczanski-Sobieski, J., Laba, K. and Kincaid, R.K. (1998) Bell-Curve Based Evolutionary Optimization Algorithm. In: Proceedings of the 7 th AIAA/USAF/NASA/ISSMO Symposium on Multidisciplinary Analysis and Optimization. (pp. 20832096) St. Lonis, MO., AIAA Paper 98-4971. 
